\title{
A preliminary study on serum liver function indices of Diethylnitrosamine induced hepatocarcinogenesis and chemoprotective potential of Eclipta alba in male Wistar rats
}

\author{
R. Anoopraj, S. Hemalatha and C. Balachandran \\ Department of Pathology, Madras Veterinary College, \\ Tamil Nadu Veterinary and Animal Sciences University (TANUVAS), Chennai- 600 007, Tamil Nadu, India \\ Corresponding author: R. Anoopraj, email: dranooprajvet@gmail.com. Present address: Division of Pathology, Indian \\ Veterinary Research Institute, Izatnagar - 243122, India. email: dranooprajvet@gmail.com \\ SH: hemacul@yahoo.com; CB: cbalachandran@yahoo.com \\ Received: 11-04-2014, Revised: 26-05-2014, Accepted: 29-05-2014, Published online: 29-06-2014
}

doi: 10.14202/vetworld.2014.439-442 How to cite this article: Anoopraj R, Hemalatha S and Balachandran C (2014) A preliminary study on serum liver function indices of Diethylnitrosamine induced hepatocarcinogenesis and chemoprotective potential of Eclipta alba in male Wistar rats, Veterinary World 7(6): 439-442.

\begin{abstract}
Aim: To study the effect of $E$. alba on liver function in experimental hepatocarcinogenesis in Wistar rats.

Materials and Methods: The study was designed to identify the protective effect of Eclipta alba in experimental diethylnitrosamine (DEN) induced hepatocarcinogenesis in rats. Sixty four male Wistar albino rats were randomly allotted to four groups of 16 rats each. DEN $(0.01 \%, \mathrm{v} / \mathrm{v})$ was given in drinking water ad libitum and E. alba $(50 \mathrm{mg} / \mathrm{kg}$ BW/day) was administered per os either alone or in combination for 120 days. Serum protein, albumin and liver functional status enzyme profiles were studied.

Results: Administration of DEN resulted in reduction in the body weights and DEN in combination with E. alba caused poor weight gain in male Wistar albino rats. Concurrent administration of $E$. alba with DEN significantly alleviated the effects of DEN on serum protein and albumin, while a partial protective effect was observed for other biochemical values and body weight.
\end{abstract}

Conclusion: The designed study could establish the partial protective effect of $E$. alba in experimentally induced hepatocarcinogenesis in rats.

Keywords: albumin, diethylnitrosamine, E. alba, hepatocarcinogenesis, liver functional status enzymes, serum protein, Wistar albino rats.

\section{Introduction}

With the advent of industrialization and modern technology, a large amount of synthetic compounds has been poured into man's biosphere as pharmaceutical products, cosmetics, food additives, agricultural chemicals etc. Most of these products gain entry into human or animal's body either deliberately or accidentally by ingestion. The liver is the first organ to be exposed to these chemicals. During this exposure, the absorbed chemicals undergo bio-transformation in the liver. In this process, liver may generate reactive or toxic metabolites and thus the liver is confronted with a higher concentration of potential toxins than any other organ in the body. Liver may respond to the bio- transformed toxic metabolites in different ways. Acute exposure to toxins results in breakdown of normal lipid metabolism, hepatocellular necrosis and/ or hepatobiliary dysfunction, while prolonged chronic exposure usually results in cirrhosis or neoplastic transformations. Tumors of liver form one of the most common neoplasms encountered and hepatocellular carcinoma (HCC) accounts for $90 \%$ of all liver tumour incidences, ranking third in cancer related deaths [1]. The possibility of their formation in

Copyright: The authors. This article is an open access article licensed under the terms of the Creative Commons Attribution License (http://creativecommons.org/licenses/by/2.0) which permits unrestricted use, distribution and reproduction in any medium, provided the work is properly cited. gastrointestinal tract makes nitrosamines one of the interesting chemicals to be studied for the various effects in the mammalian body system. They are formed by the nitrosation of simple amine precursors in mammalian organism. The precursors of nitrosamines are almost ubiquitous in the environment, as they are contained in numerous foods, beverages, cosmetics, and medicines. The best studied nitrosamines like tobacco specific nitrosamines, dimethylnitrosamine, and diethylnitrosamine possess potential toxic and carcinogenic properties. Diethylnitrosamine (N-nitrosodiethylamine, DEN) is a well known hepatotoxin as well as hepatocarcinogen found in air, water and soil, and is normally used as a carcinogen to induce liver cancer in animal models [2, 3, 4]. Metabolic activation of DEN in liver produces ethylating electrophiles which react with DNA resulting in the formation of DNA adducts [5].

Recent advanced researches aiming to control malignancy by pharmacological intervention have reached new heights. Many synthetic and natural compounds have been discovered which can either arrest or reverse the process of malignancy significantly. Eclipta alba is an annual shrub which has been reported to be useful in liver ailments. It has been proved that $E$. alba possesses antioxidant as well as free radical scavenging properties. Moreover, it can 
Table- 1: Body weight of control and treated animals (mean $\pm \mathrm{SE})$.

\begin{tabular}{lcc}
\hline Groups & Initial body weights $\mathbf{( g )}$ & Final body weights $\mathbf{( g )}$ \\
\hline Control & $152.13 \pm 3.78$ & $212.00^{\mathrm{c}} \pm 8.34$ \\
DEN & $152.13 \pm 3.19$ & $92.00^{\mathrm{a}} \pm 13.32$ \\
E. alba & $152.00 \pm 3.16$ & $216.33^{\mathrm{c}} \pm 15.62$ \\
DEN+E.alba & $152.38 \pm 4.14$ & $175.67^{\mathrm{b}} \pm 4.33$ \\
\hline
\end{tabular}

$P<0.05$, means with same superscript within a column do not differ from each other.

correct altered activity of $\mathrm{Na}^{+}, \mathrm{K}^{+}$and ATPase associated with toxic injury [6].

Studies have confirmed its hepatoprotective activities against carbon tetrachloride and paracetamol induced hepatocyte damage in animals [7, 8, 9]. But a systematic research on any possible effect of $E$. alba on hepatocarcinogenesis seems to be scarce or absent [10]. In view of the above facts, this study investigated the protective effect of $E$. alba in experimentally DEN induced hepatocarcinogenesis in rats.

\section{Materials and Methods}

Ethical approval: The present study was conducted at the Department of Veterinary Pathology, Madras Veterinary College, Chennai. This study was approved by the Institutional Animal Ethics Committee.

Animals: Male albino rats (Wistar strain) of 7-9 weeks age weighing around 120-150 g obtained from Department of Laboratory Animal Medicine, Centre for Animal Health Studies, Tamil Nadu Veterinary and Animal Sciences University (TANUVAS) Madhavaram Milk Colony, Chennai, were used for the study. The animals were housed in polypropylene cages at a population density of six per cage. Animals were acclimatized for two weeks at Centralized Laboratory Animal House, Madras Veterinary College, with normal light and dark cycle. A commercial rat pellet $\operatorname{diet}(\mathrm{M} / \mathrm{s}$ Vet Care Ltd., Bangalore, India) and water were provided ad libitum.

Experimental protocol: Sixty four male albino rats of Wistar strain were weighed and randomly distributed into four groups, each group comprising of 16 rats. Group I consisted of normal control rats, maintained on commercial rat pellet diet and water; Group II, received $0.01 \%$ DEN (v/v) (Isopac ${ }^{\circledR}, 1 \mathrm{~g}$, Sigma Chemical Co. USA) in drinking water ad libitum for 120 days to induce hepatocarcinogenesis; Group III, treated with E. alba $(50 \mathrm{mg} / \mathrm{kg} \mathrm{BW/day} \mathrm{p.o} \mathrm{(per} \mathrm{os))} \mathrm{(Indian} \mathrm{Herbs}$ Research \& Supply Co. Ltd, Saharanpur, India) in distilled water; Group IV, treated with $0.01 \%$ DEN $(\mathrm{v} / \mathrm{v})$ in drinking water ad libitum and Eclipta alba $(50$ $\mathrm{mg} / \mathrm{kg} \mathrm{BW} /$ day p.o) in distilled water simultaneously for 120 days. Animals were sacrificed at the end of experiment. The blood samples were collected for biochemical analysis and were allowed to clot and centrifuged at $1500 \mathrm{rpm}$ for $20 \mathrm{~min}$ to separate the sera. Serum total protein and albumin were estimated by modified Biuret and Dumas method, aspartate
Table-2: Effect of E.alba on serum total protein, albumin and globulin of control and treated animals (mean $\pm S E$ ).

\begin{tabular}{|c|c|c|c|}
\hline Groups & Total protein $(\mathbf{g} / \mathrm{dL})$ & Albumin (g/dL) & Globulin (g/dL) \\
\hline Control & $6.70^{b} \pm 0.23$ & $2.46^{b} \pm 0.03$ & $4.06 \pm 0.27$ \\
\hline DEN & $5.40^{a} \pm 0.27$ & $1.75^{\mathrm{a}} \pm 0.14$ & $3.65 \pm 0.31$ \\
\hline E.alba & $6.83^{b} \pm 0.23$ & $2.51^{b} \pm 0.08$ & $4.32 \pm 0.25$ \\
\hline $\mathrm{DEN}+$ E.alba & $6.36^{b} \pm 0.21$ & $2.43^{\mathrm{b}} \pm 0.10$ & $3.94 \pm 0.25$ \\
\hline
\end{tabular}

$\mathrm{P}<0.05$, means with same superscript within a column do not differ from each other.

aminotransferase (AST), alanine aminotransferase (ALT) and alkaline phosphatase (ALP) by IFCC (International Federation of Clinical Chemistry) method, gammaglutamyl transferase (GGT) by Szasz method.

Histopathological study: Representative liver samples from each group were collected in 10\% neutral buffered formalin. These samples were processed and stained by routine H\&E stain for histopathological examinations following standard protocol.

Statistical analysis: The data obtained from different parameters were subjected to one-way analysis of variance using SPSS (Version 10.0 for windows) statistical software.

\section{Results}

The mean $( \pm \mathrm{SE})$ values of body weights of adult male rats treated with DEN, DEN+E.alba and E.alba are presented in Table-1. Comparison of mean body weights revealed that there was no significant difference between the control and the E. alba group. In DEN group animals, there was a significant decrease in the final body weight when compared with control, $E$. alba and $\mathrm{DEN}+$ E.alba. The $\mathrm{DEN}+$ E.alba animals showed a significant increase in the final body weight when compared with DEN treated animals and a significant decrease when compared with control and E. alba groups.

The mean $( \pm \mathrm{SE})$ values of total protein, albumin and globulin of adult male rats treated with DEN, DEN $+E$. alba and E. alba are given in Table-2. There was a significant decrease in the serum total protein and albumin in the DEN group when compared to the other groups. No significant difference was observed among the control, DEN, E. alba, DEN+E.alba groups for serum globulin. Table-3 shows mean $( \pm \mathrm{SE})$ serum ALT, AST, ALP and GGT values in adult male rats of control, DEN, E. alba and DEN+E.alba groups. There was a significant increase in the levels of ALT, ALP and GGT in the DEN group when compared to the other groups. Significant decrease in the ALT, ALP and GGT values were observed in the $\mathrm{DEN}+$ E. alba group when compared with the DEN alone group. But there was a significant increase in the levels of serum ALT, AST and GGT in the DEN+E. alba treated groups when compared with control and $E$. alba alone groups.

Histopathological examinations revealed normal hepatic architecture of liver sections from control and E. alba alone group (Figure-1). Sections of liver from 
Table-3: Effect of E. alba on serum ALT, AST, ALP and GGT of control and treated animals (mean \pm SE).

\begin{tabular}{|c|c|c|c|c|}
\hline Groups & ALT (U/L) & AST (U/L) & ALP (U/L) & GGT (U/L) \\
\hline Control & $64.50^{\mathrm{a}} \pm 2.89$ & $213.79 \pm 7.31$ & $841.25^{a} \pm 20.16$ & $2.58^{a} \pm 0.24$ \\
\hline DEN & $142.60^{\mathrm{C}} \pm 11.62$ & $250.47 \pm 7.61$ & $1502.59^{b} \pm 181.34$ & $31.69^{c} \pm 4.32$ \\
\hline E.alba & $64.17^{a} \pm 4.86$ & $203.75^{a} \pm 9.41$ & $871.84^{a} \pm 21.81$ & $2.72^{a} \pm 0.33$ \\
\hline DEN+E.alba & $105.81^{b} \pm 14.13$ & $266.45^{b} \pm 6.07$ & $1053.66^{a} \pm 104.41$ & $11.69^{b} \pm 4.37$ \\
\hline
\end{tabular}

$\mathrm{P}<0.05$, means with same superscript within a column do not differ from each other.

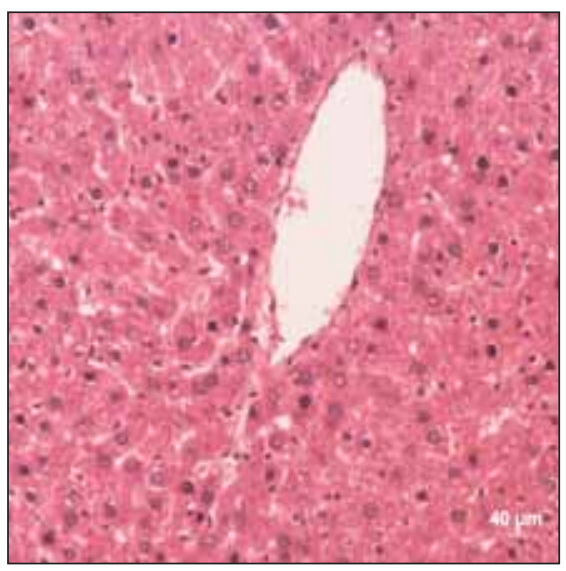

Figure-1: Liver-normal architecture, $\mathrm{H}$ \& $\mathrm{Bar}=40 \mu$.

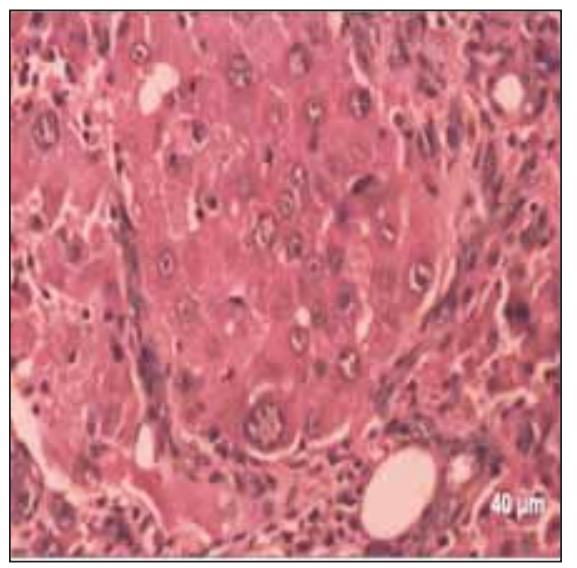

Figure-2: Neoplastic nodule with pleomorphic hepatocytes, atypical mitosis and atrophy of lining hepatocytes, H\&E Bar $=40 \mu$.

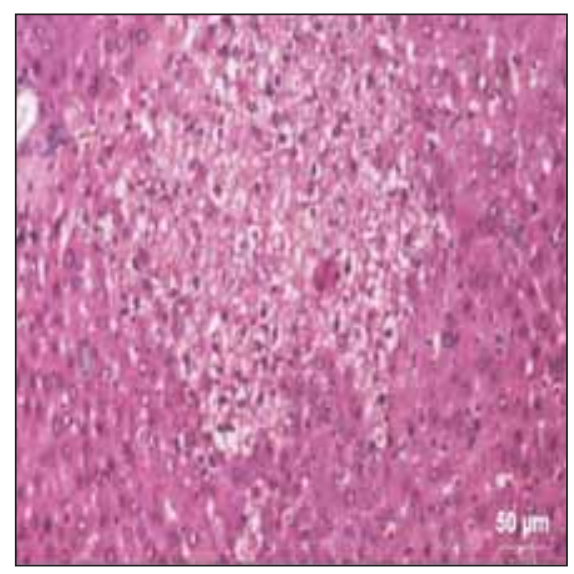

Figure-3: Clear cell foci in liver, H\&E Bar $=50 \mu$.
DEN treated group consistantly showed characteristic hepatocellular carcinoma in all animals, with loss of normal architecture, pleomorphic hepatocytes forming neoplastic nodule, atypical mitosis and pressure atrophy of adjacent peripheral hepatocytes (Figure-2). Microscopical examinations of liver sections from DEN + E. alba showed a variation in histopathological picture. It varied from formation of clear cell foci (Figure-3), which was an early change in hepatocarcinogenesis (in $70 \%$ of animals) to the presence of hepatocellular carcinoma (in the rest $30 \%$ of animals).

\section{Discussion}

Treatment with DEN caused significant reduction in body weight of adult male rats, while poor weight gain was observed in the DEN+E.alba treated rats. However, the treatment with E. alba improved the weight gain by $39 \%$ when compared to the DEN alone treated rats. This clearly indicated that $E$. alba had a protective role over the DEN induced reduction in body weight gain of rats. Earlier studies [11, 12] also showed a significant reduction in body weight of rats treated with DEN after 11 weeks. The loss of weight induced by DEN is plausible because this compound specifically damages the liver which may result in the derangement of energy metabolism. The protective effect of E. alba on DEN induced reduction in body weights noticed in the present study could be attributed to the free radical scavenging activity of the E. alba, thus protecting the hepatocytes from free radical induced injury [6].

Reduction in serum total protein and albumin in DEN treated group could be ascribed to the develop- ment of hepatic lesions observed in the present study that affected protein synthesis, which concurred with earlier findings [13]. The E. alba treatment protected the liver from the effects of DEN on serum protein and albumin. Significant increase in the ALT, ALP and GGT values were observed in the DEN treated group, when compared to the other groups. These findings are in accordance with the earlier reports of significant increase in ALT, AST and GGT values in rats fed with 0.01 percent DEN in drinking water ad libitum for 15 weeks [12]. Similar observations were made for ALT, ALP and GGT in rats dosed $10 \mathrm{mg}$ DEN/ $\mathrm{kg} \mathrm{BW}$, five times a week for 15 weeks intragastrically [14]. The increase in the activities of serum ALT, ALP and AST occurred when hepatocellular damage caused abnormalities of liver function, and serum concentrations of these enzymes increased remarkably in hepatoma [12]. There was a 15 fold increase in the serum GGT values in the DEN treated rats when compared to the control rats, which was more than double fold increase in the serum GGT values recorded in previous studies. The GGT level increased with the emergence of neoplastic nodules and retained during differentiation of hepatoma. The elevated GGT level enabled the cells to respond to the proliferation by other stimuli and might facilitate nodule formation and tumour progression [15]. The increased activities of serum ALT, ALP, GGT and AST in the present study could be attributed to the hepatic lesions produced by DEN. Serum GGT levels of animals in $\mathrm{DEN}+$ E. alba group showed only five fold increase, which was well below the 15 fold increase encountered in DEN alone treated group. The significant increase in the serum ALT, GGT and AST values in the 
$\mathrm{DEN}+$ E.alba group when compared to the control and E. alba alone treated rats and the significant decrease in the serum ALT and GGT values when compared to the DEN alone treated rats reflect the partial protective effect of E. alba on DEN induced hepatic lesion [16].

\section{Conclusion}

E. alba is one of the important ingredients of hepatoprotective ayurvedic formulations. The designed experiment elucidated the effect of this annual shrub on hepatocarcinogenesis. Eventhough the treatment with $E$. alba couldn't protect the liver completely, the present study revealed a partial protective effect of $E$. alba on DEN induced hepatocarcinogenesis as indicated by histopathological examinations and values of liver function indices. This can be attributed to the protective effect of $E$. alba on DEN induced oxidative cell membrane damage.

\section{Authors' contributions}

$\mathrm{RA}, \mathrm{SH}$, and CB designed the experiment. RA conducted the research experiment. $\mathrm{RA}, \mathrm{SH}$, and $\mathrm{CB}$ drafted and revised the manuscript. All authors read and approved the final manuscript.

\section{Acknowledgements}

The authors are highly thankful to The Dean, Madras Veterinary College, Chennai for providing the facilities to carry out the study. Financial assistance from Indian Council of Agricultural Research provided to the first author (RA) in the form of Junior Research Fellowship is also gratefully acknowledged.

\section{Competing interests}

The authors declare that they have no competing interests.

\section{References}

1. Herszenyi, L. and Tulassay, Z. (2010) Epidemiology of gastrointestinal and liver tumors. Eur. Rev. Med. Pharmacol. Sci., 14:249-258.

2. Salim, S.A.R., Abdulaziz, M.A., Abdulaziz, A. A.Y., Saleh, A. B., Abdulmalik, A., Amal, G. F., Othman, A.A.S. and Mohamed, M.S.A. (2009) Progression of diethylnitrosamine-induced hepatic carcinogenesis in carnitine-depleted rats. World J. Gastroenterol., 15: 1373-1380.
3. Thorsten, M., Florian, R.T., Amrit, M., Thomas, L., Peter, S., Dennis, S., Torsten, H., Peter, R. G., Andreas, T. and Stephan, K. (2011) Liver specific overexpression of platelet-derived growth factor- $\beta$ accelerates liver cancer development in chemically induced liver carcinogenesis. Int. J. Canc., 128: 1259-1268.

4. Guo, C., Zhi-Kai, D., Rong-Gan, L., Sheng-Jun, X., SongQing, H., Hai-Lu, Z. and Qing, X. (2012) Characterization of diethylnitrosamine-induced liver carcinogenesis in Syrian golden hamsters. Exp. Ther. Med., 3: 285-292.

5. Wu, L., Tang, Z.Y. and Li, Y. (2009) Experimental models of hepatocellular carcinoma: Developments and evolution. $J$. Canc. Res. Clin. Oncol., 135: 969-981.

6. Majumdar, A.S., Saraf, M.N., Andrades, N.R. and Kamble, R. (2008) Preliminary studies on the antioxidant activity of Tribulus terrestris and Eclipta alba. Phcog. Mag., 4: 102-107.

7. Samudram, P., Hari, R., Vasuki, R., Geetha, A. and Sathiya Moorthi, P. (2008) Hepatoprotective activity of Bi-herbal ethanolic extract on CCl4 induced hepatic damage in rats. Afr. J. Biochem.Res., 2: 61-65.

8. Lima, T.B., Suja, A., Jisa, O.S., Sathyanarayanan, S. and Remya, K. S. (2010) Hepatoprotective activity of LIV-first against carbon tetra chloride-induced hepatotoxicity in albino rats. Int. J. Green. Pharm., 4: 71-74.

9. Prabhu, K., Kanchana, N and Sadique, A.M. (2011) Hepatoprotective effect of Eclipta alba on paracetamol induced liver toxicity in rats. J. Microbiol. Biotechnol. Res., 1:75-79.

10. Harshita, C., Vikram, D., Jatinder, S., Sukhdev, S.K. and Sriram, S. (2011) Evaluation of hydro-alcoholic extract of Eclipta alba for its anticancer potential: An in vitro study. $J$. Ethnopharmacol., 136:363-367.

11. Ramakrishnan, G., Raghavendran, H.R.B., Vinodhkumar, R. and Devaki, T. (2006) Supression of N-nitrosodiethylamine induced hepatocarcinogenesis by silymarin in rats. Chem. Biol. Interact., 161: 104-114.

12. Kim, C.H., Moon, P.I. and Kang, C.B. (1999) Changes on function and morphology of liver in carcinogen induced hepatoma rats. Korean J. Vet. Serv., 22: 61-70.

13. Ha, W.S., Kim, C., Song, S. and Kang, C. (2001) Study on mechanism of multistep hepatotumorigenesis in rat: Development of hepatotumorigenesis. J. Vet. Sci., 2: 53-58.

14. Sadik, N.A.H., Maraghy, S. A and Ismail, M.F. (2008) Diethylnitrosamine-induced hepatocarcinogenesis in rats: Possible chemoprevention by blueberries. Afr. J. Biochem. Res., 2: 81-87.

15. Hanigan, M.H. and Pitot, H.C. (1985) Gamma-glutamyl transpeptidase-its role in hepatocarcinogenesis. Carcinogenesis, 6: 165-172.

16. Lin, S.C., Yao, C.J., Lin, C.C. and Lin, Y.H. (1996). Hepatoprotective activity of Taiwan folk medicine: Eclipta prostrata against various hepatotoxins induced acute hepatotoxicity. Phytother. Res., 10: 483-490. 